\title{
DDS Perspective: My Take on Therapeutic Drug Monitoring in IBD
}

\author{
Maria T. Abreu ${ }^{1}$ \\ Published online: 18 October 2019 \\ (c) Springer Science+Business Media, LLC, part of Springer Nature 2019
}

Let me explain the impetus for writing this perspective piece. First, my gastroenterology fellowship coincided with the dawn of the new day of biologics [1]. The treatment of inflammatory bowel disease (IBD) can be divided into "before infliximab" and "after infliximab." Until that time, we did not discuss mucosal healing as an endpoint. We were content with symptomatic improvement for our patients. As such, I have lived the learning curve of anti-tumor necrosis factor (TNF) antibody ( $\mathrm{Ab}$ ) use and, more recently, ustekinumab and vedolizumab (for now). Alongside the availability of biologics, tests to measure serum levels of the biologics and Abs to the biologic are commercially available. In this perspective piece, I will attempt to synthesize the history of therapeutic drug monitoring (TDM) and how I use it in my very tertiary referral practice. The other reason for the timing of this article is the relatively recent publication of the American Gastroenterological Association (AGA) guidelines for TDM. The authors do an outstanding job of rating the literature and providing recommendations based on their interpretation of the available data. I have the opportunity to provide a different perspective on the same data. Thus, I hope to combine experience and evidence for my recommendations on TDM.

\section{Immunogenicity and Biologics}

For the purposes of this article, biologics refer to monoclonal Abs used in the treatment of IBD. All of these monoclonal Abs are synthetically made proteins. Most are generated by taking the DNA sequence for the heavy and light chains of the $\mathrm{Ab}$ and introducing it into a cell line that serves as the factory for its synthesis and glycosylation. All of these

Maria T. Abreu

MAbreu1@med.miami.edu

1 Division of Gastroenterology, Crohn's and Colitis Center, University of Miami Miller School of Medicine, 1011 NW 15th Street, Gautier, Suite 510 (D-149), Miami, FL 33136, USA proteins have the potential for causing immunogenicity, although there are clear differences in immunogenicity with newer biologics. In the earliest days of infliximab, the issue of immunogenicity was striking. Patients in the original clinical trial of cA2 (infliximab) often received only one dose of the medication or only a few doses of the medication followed by years of waiting for U.S. Food and Drug Administration (FDA) approval. Likewise, when infliximab was first approved, it was only approved for active disease, and thus patients routinely had dose interruptions. When these patients were re-dosed with infliximab, most developed a delayed-type hypersensitivity reaction. Unfortunately, we inadvertently immunized patients to the infliximab.

Fundamentally, there are two types of infusion reactions mediated by antidrug Abs-immediate-type and delayedtype hypersensitivity (DTH) reactions. The immediate-type hypersensitivity is manifested by shortness of breath and flushing, although thankfully anaphylaxis is extraordinarily rare. This reaction is usually dealt with antihistamines and intravenous (IV) glucocorticoids at the time of the infusion. DTH reactions typically occur within 3-7 days after an infusion and are manifested by a migratory polyarthritis. Often patients have jaw pain, which is characteristic of this DTH reaction. Typically, patients receive the first infusion after a dose interruption uneventfully, but it is usually with the second dose after a dose interruption that patients develop either the immediate-type hypersensitivity reaction or DTH reaction. DTH is treated with a methylprednisolone pack or a short course of glucocorticoids. At the end of it all, whether it is an immediate-type hypersensitivity reaction or a DTH reaction to infliximab, patients can no longer receive infliximab.

The first publication that systematically looked at Abs against infliximab came from the Belgian Group [2]. They showed that patients in whom Abs to infliximab developed had a much shorter response to the infliximab and had much lower serum levels of infliximab. It all seems obvious to us now, but that was an important observation. Since these early days, we have learned, and are still learning, how best to prevent this from happening. The Belgian Group showed early 
on that those patients who were on thiopurines were less likely to have developed Abs to the infliximab. This has led us to a cottage industry of how to deal with immunogenicity.

We also know that adalimumab causes immunogenicity; it does so to a lesser extent than infliximab [3]. The immunogenicity with adalimumab can be a great deal more subtle than that with infliximab because it will not cause an immediate-type hypersensitivity reaction. Because adalimumab is administered subcutaneously, the drug is released more slowly into the systemic circulation and does not cause immune complex formation. Manifestations of Abs against adalimumab can range from just a loss of response to the medication to a polyarthropathy or a rash with detectable Abs to adalimumab.

\section{Avoiding Immunogenicity}

Early attempts to avoid immunogenicity included administration of a dose of IV glucocorticoids before each dose of infliximab [4]. What turns out to be a more effective strategy is to administer infliximab or adalimumab with a thiopurine or methotrexate. The combination of a thiopurine or methotrexate with infliximab or adalimumab tends to raise the level of the biologic, especially infliximab. Our group has demonstrated that one does not need to achieve high levels of the thiopurine in order to have this effect and that levels of thioguanine of $125 \mathrm{pmol} / 8 \times 10^{8}$ or greater can increase the levels of the infliximab [5]. Likewise, David Rubin's group has shown that methotrexate doses of $12.5 \mathrm{mg}$ per week and above, generally given orally, are sufficient to prevent immunogenicity and raise the serum levels of the biologic [6]. I view immunogenicity and low drug levels as being interconnected, since accelerated clearance of the biologics is due to Abs against these biologics.

Newer biologics such as ustekinumab and vedolizumab are associated with very low rates of immunogenicity. Ustekinumab is the one with the very lowest rate, with less than 5\% of patients developing Abs to ustekinumab [7]. It is not entirely clear why these newer biologics do not lead to immunogenicity. My own working hypothesis is that there has been a tremendous improvement in manufacturing processes with a reduction in contaminates from the cell lines that serve as adjuvants in the immune response. This raises a whole other issue, which is whether or not combination therapy is necessary for these new biologics. We know that, at least for ustekinumab, the addition of an immunomodulator does not alter the serum levels of the drug [8].

Who is at risk for developing Abs against the biologics? There are known risks for increased clearance of proteinbased monoclonal Abs, including a high inflammatory burden, which may be manifest by a high C-reactive protein or fecal calprotectin level, a low serum albumin level, and extensive colitis. Recently, the HLA-DQA $1 * 05$ haplotype was identified as a genetic determinant of immunogenicity to TNF antagonists. Patients with the HLA-DQA $1 * 05$ haplotype have a twofold increased risk of immunogenicity to infliximab or adalimumab [3]. Approximately $40 \%$ of Europeans carry the HLA-DQA $1 * 05$ haplotype. In patients on infliximab monotherapy carrying this allele, $92 \%$ had Abs to infliximab by one year. Ideally, one would develop a composite score with these clinical and genetic variables to determine a patient's individual risk of developing immunogenicity.

Biosimilars are no different. By their very design, they are meant to be identical to the parent drug. If patients have already become immunized to infliximab, then there is no role to switching them to a biosimilar of infliximab. All the things that we have painstakingly learned over a long period of time must still be kept in mind. For the foreseeable future, biosimilars to infliximab and adalimumab will be the first choice by insurance companies, because of their lower cost.

What happens once people have developed immunogenicity to an anti-TNF? A sobering recent study has shown that for patients who have developed Abs to infliximab and are changed to adalimumab, although in the short-term the adalimumab is effective, by one year patients already have lost the effectiveness of the adalimumab as well [9]. By contrast, those patients who had low levels of infliximab in whom the dose is raised seem to do well. This finding has led to the speculation that with the second anti-TNF, one should absolutely use an immunomodulator to prevent immunogenicity. On the other hand, this recommendation has not been tested prospectively. Even in patients with the HLA-DQA $1 * 05$ haplotype, the risk of immunogenicity is halved when the biologic is used in combination with an immunomodulator.

Once a patient has developed Abs to infliximab or adalimumab, things can be done to regain response and suppress these Abs [10]. I use as a guiding principal whether patients have remaining levels of the biologic at trough or not. If the patient has no remaining drug level at trough and has Abs to the drug, the game is over, and there is no point in trying to continue the biologic. On the other hand, if the patient has low levels of Abs against the biologic and is still having a clinical effect after a dose of the drug, it is worth attempting more frequent dosing, higher doses, and the addition of an immunomodulator. When performing TDM, you will notice that the levels of the Abs against the biologic are reported on a different scale than the levels of the biologic itself.

\section{Loss of Response}

With any of these medications, there are patients who will not initially respond. The most recent relevant large study has found that about a quarter of patients fall into this category 
[11]. No genetic or other biomarkers have been found to be useful for making clinical decisions about who is less likely to respond to an anti-TNF. What is far more common is for patients to have a partial response. For both adalimumab and infliximab, and possibly with all the other biologics, this initial (or chronic) partial response may very well be due to insufficient serum levels. Therefore, I am a believer that, early on, pushing the levels of the medication is a strategy worth trying. This is particularly true for those patients who have a high inflammatory burden or fistulizing disease. We have reported that patients with fistulizing disease need much higher levels of the drug in order to have a benefit [12]. The mean level of infliximab at trough in patients with fistula healing was $16 \mu \mathrm{g} /$ $\mathrm{mL}$ (based on the Prometheus assay). The other important experiment we performed was to measure levels of infliximab and adalimumab in intestinal tissue [13]. Even when patients have reasonably good systemic levels of the biologic in tissue, there can be high local production of TNF and other effector cytokines that continue to drive inflammation.

For patients who have lost response or are losing response, measuring levels of the drug of course can be an important maneuver. Several studies have shown that patients with low levels of the drug respond well to an increase in the dose. However, there is another group of patients who seem to be increasing in number, at least in referral centers, who lose the response even in the absence of developing Abs and with "adequate" drug levels. In these latter patients, the more likely scenario is that they have had a mechanistic loss of response, and more anti-TNF is unlikely to lead to a durable response.

At present, the response to the first biologic (or small molecule) is better than the response to subsequent treatmentwhether or not the treatment is another anti-TNF or another class of biologic. For this reason, I am a believer in optimizing the first biologic, generally an anti-TNF, as long as possible. For example, in the subgroup of patients who were on a previous anti-TNF, the effect of ustekinumab barely reached statistical significance [14]. So far, only tofacitinib in a network meta-analysis has been demonstrated to be effective after an anti-TNF. Even with vedolizumab, the subgroup of patients who have been on an anti-TNF previously has a substantial drop-off in responsiveness compared with biologic-naïve patients [15]. As a rule, patients in whom you are considering a change in biologic should be evaluated to make sure that there are (1) active inflammation and (2) no other complication such as a stricture that will never respond to any medical therapy we currently have.

\section{What Drug Levels Does One Need to Achieve?}

Here is the trickiest part of the entire story. Wouldn't it be great if I could give you an actual level that one needs to achieve, that cuts across all the biologics so you only had to remember one number? I wish I could. I think the number 10 is probably a good number for anti-TNFs. I think that, regardless of the assay, levels greater than $10 \mu \mathrm{g} / \mathrm{mL}$ for both adalimumab and infliximab probably capture patients at every end of the spectrum. I do think that therapeutic drug levels are a little bit like ammonia levels, that they depend on the patient and the scenario. The actual numerical value also depends on the laboratory that is being used. In general, I recommend that clinicians become used to a particular laboratory and how it reports levels and that they use the same company each time they check a particular patient's levels so that one has an idea of the operating characteristics for that particular patient.

As I mentioned earlier, our own studies have shown that levels of infliximab have to be higher in patients who have fistulizing disease in order to see an improvement in fistulas. Although we only studied this for infliximab, I think we can apply the same principals to the other anti-TNFs and possibly even to ustekinumab, which is a second-line agent in patients who have fistulizing disease. For patients who have extensive severe colitis, whether Crohn's colitis or ulcerative colitis, higher levels are needed in order to achieve the tissue levels that are necessary to induce mucosal healing. However, once the bowel is healed, the levels probably do not need to be as high. For patients who are doing well, I do not necessarily raise the dose if the levels of the biologic are above $5 \mu \mathrm{g} / \mathrm{mL}$.

As with all of the studies of biologics, if one looks at the post hoc analysis of patients on an immunomodulator with the biologic, there does not appear to be an increased benefit in patients who are on an immunomodulator with vedolizumab or ustekinumab. I will remind the readers, however, that until the SONIC (Study of Biologic and Immunomodulator Naïve Patients in Crohn's Disease) study [16] and then the UC SUCCESS study [17] were completed, we did not think that an immunomodulator was needed to be combined with infliximab for the treatment of Crohn's and ulcerative colitis, respectively. Only in the SONIC study did we see that the combination of infliximab with azathioprine was superior at inducing mucosal healing. More recently, we learned that patients who had high trough levels of infliximab did not seem to have an additional benefit with the addition of azathioprine [18]; therefore, there exists the possibility that the main effect of combining azathioprine or another immunomodulator with infliximab is due to the higher levels of infliximab that can be achieved. 
I do think, however, that there are patients who need an immunomodulator to help with efficacy, not simply to improve serum levels of the biologic, but also to mechanistically complement the biologic. For this assertion, I have no data other than experience with tertiary referral patients who need everything plus the kitchen sink to have a clinical benefit. Thus, although I think that it is true that, in general, we do not need an immunomodulator for vedolizumab and ustekinumab, because they are far less immunogenic than anti-TNFs, I suspect that there will be a subset of patients who could benefit from combination therapy simply because each of these drugs has a benefit on its own.

\section{Proactive Versus Reactive Testing}

I am a believer in proactive testing. If I only had one trough level to check, it would be early on, just after induction and before maintenance with any of the biologics. It does not make sense to me to wait until someone is failing to realize that levels are too low or, worse, allow patients to make Abs to the biologic. The TAXIT (Trough Concentration Adapted Infliximab Treatment) and Tailorix (Tailored Treatment with Infliximab for Active Crohn's Disease) studies are cited as evidence that proactive testing is not beneficial $[19,20]$. The study shows that an early trough level and readjustment of the dose is a highly effective strategy for long-term efficacy. I also believe that the highly trained gastroenterologists in this study brought to bear a lot of experience in adjusting the dose based on clinical symptoms. I generally check trough levels of infliximab before the first maintenance dose and, for adalimumab, in the 10- to 14-week window. For ustekinumab, I check the level prior to the second subcutaneous dose, and for vedolizumab, I check the level prior to the first maintenance dose. For vedolizumab, I like to see levels above $15 \mu \mathrm{g} / \mathrm{mL}$ at that stage. For ustekinumab, there is much variability in the levels with respect to the laboratory used. I would say that if the Prometheus laboratory is used, the levels should be greater than $8 \mu \mathrm{g} / \mathrm{mL}$, and if another laboratory is used, the level needs to be greater than $4.5 \mu \mathrm{g} /$ $\mathrm{mL}$ [21].

I could go into all the data supporting proactive testing. I acknowledge that the studies done by Adam Cheifitz and colleagues are observational [22]. Nevertheless, the results confirm my bias that if one checks levels on a regular basis, it is far more likely that you can maintain a patient well and adjust levels prior to a loss of response. Once a patient loses response and has low levels of the drug, there is a risk of developing Abs to the drug, with an inability to be rescued with any other intervention.

Much of the debate has been predicated on the fact that drug level testing can be expensive. I am a firm believer that such testing needs to be made as inexpensive as possible so we may check levels as we would order other routine laboratory tests. In general, I check levels about every 6 months in patients who are doing well. I think that even once a year is probably adequate in patients who are doing well.

\section{Side Effects and Relationship to Levels}

Higher levels of a biologic are better up to a point. In rheumatoid arthritis, giving higher doses of anti-TNFs is associated with a higher risk of infection. In IBD, higher trough levels are not associated with an increased risk of infections [22]. For biologics that appear to be safer overall, like vedolizumab and ustekinumab, the infection rate is low, and, therefore, it will be hard to show that higher levels are associated with an increased risk of infection. The aspirational goal of complete mucosal healing is just that, aspirational. It cannot be achieved in all patients, and at every point along the way we need to make decisions about whether it is worth the potential for side effects by increasing the dose of a medication or adding another drug such as an immunomodulator.

The psoriasiform reaction that we see with anti-TNFs is common if you are looking for it. Patients develop a rash either on their hands and feet, which can be painful, behind the ears, and on their scalp. Interestingly, there are still dermatologists who do not realize that this is a psoriasiform reaction to the anti-TNF and tell patients that it has nothing to do with the anti-TNF; but we as gastroenterologists know better. Levels of the anti-TNF have not been clearly linked to the psoriasiform reaction [23]; it is likely to do with unopposed action of interferon-alpha [24]. Nevertheless, I would suggest reevaluating trough levels of the drug and adjusting the dose downward if there is room to do so. You may even have to stop the concurrent immunomodulator and let the biologic level drift down. Although ustekinumab is approved for the treatment of psoriasis and can be highly effective in traditional psoriasis, it is not nearly as effective in patients who have a psoriasiform reaction. Probably the most effective approach is simply to change the patient to something other than an anti-TNF, and over time the psoriasiform reaction will simply resolve.

\section{Summary}

I hope I have imparted some of what I have learned over decades of using biologic medications. The story is nowhere near over. Whether we are giving a biologic agent or small molecule, I think we need to keep an open mind that testing of drug levels or performing functional assays should guide us with respect to dosing. I think this is not as simple as managing high blood pressure, where one can easily check the blood pressure. In a patient with IBD, we cannot 
do repeated real-time endoscopy. I am looking forward to a rich future of having many new delivery mechanisms and types of medications available for patients with IBD with each medication coming with a companion diagnostic test to predict efficacy.

\section{Guiding Principles}

- It is never as good as the first time- -be proactive to maximize the benefit of the first biologic therapy a patient receives.

- Newer biologics are less immunogenic; thus, the need for concurrent immunomodulators should be driven by the need for efficacy rather than the prevention of immunogenicity.

- Higher levels of biologics are needed for the "bigger" problems-extensive colitis, deep ulcers, and fistulizing disease.

\section{References}

1. Targan SR, Hanauer SB, van Deventer SJ, et al. A short-term study of chimeric monoclonal antibody cA2 to tumor necrosis factor alpha for Crohn's disease. Crohn's Disease cA2 Study Group. N Engl J Med. 1997;337:1029-1035.

2. Baert F, Noman M, Vermeire S, et al. Influence of immunogenicity on the long-term efficacy of infliximab in Crohn's disease. $N$ Engl J Med. 2003;348:601-608.

3. Sazonovs A, Kennedy N, Moutsianas L, et al. HLA-DQA1*05 is associated with the development of antibodies to anti-TNF therapy. bioRxiv. 2018:410035.

4. Farrell RJ, Alsahli M, Jeen YT, Falchuk KR, Peppercorn MA, Michetti P. Intravenous hydrocortisone premedication reduces antibodies to infliximab in Crohn's disease: a randomized controlled trial. Gastroenterology. 2003;124:917-924.

5. Yarur AJ, Kubiliun MJ, Czul F, et al. Concentrations of 6-thioguanine nucleotide correlate with trough levels of infliximab in patients with inflammatory bowel disease on combination therapy. Clin Gastroenterol Hepatol. 2015;13:1118-1124.

6. Colman RJ, Rubin DT. Optimal doses of methotrexate combined with anti-TNF therapy to maintain clinical remission in inflammatory bowel disease. J Crohns Colitis. 2015;9:312-317.

7. Hanauer SB, Sandborn WJ, Feagan BG, et al. IM-UNITI: 3 year efficacy, safety, and immunogenicity of ustekinumab treatment of Crohn's disease. J Crohns Colitis. Epub. 06/03/2019.

8. Adedokun OJ, Xu Z, Gasink C, et al. Pharmacokinetics and exposure response relationships of ustekinumab in patients with Crohn's disease. Gastroenterology. 2018;154:1660-1671.

9. Roblin X, Verot C, Paul S, et al. Is the pharmacokinetic profile of a first anti-TNF predictive of the clinical outcome and pharmacokinetics of a second anti-TNF? Inflamm Bowel Dis. Epub. 04/26/2018.

10. Ungar B, Kopylov U, Engel T, et al. Addition of an immunomodulator can reverse antibody formation and loss of response in patients treated with adalimumab. Aliment Pharmacol Ther. 2017;45:276-282.

11. Kennedy NA, Heap GA, Green HD, et al. Predictors of anti-TNF treatment failure in anti-TNF-naive patients with active luminal Crohn's disease: a prospective, multicentre, cohort study. Lancet Gastroenterol Hepatol. 2019;4:341-353.

12. Yarur AJ, Kanagala V, Stein DJ, et al. Higher infliximab trough levels are associated with perianal fistula healing in patients with Crohn's disease. Aliment Pharmacol Ther. 2017;45:933-940.

13. Yarur AJ, Jain A, Sussman DA, et al. The association of tissue anti-TNF drug levels with serological and endoscopic disease activity in inflammatory bowel disease: the ATLAS study. Gut. 2016;65:249-255.

14. Feagan BG, Sandborn WJ, Gasink C, et al. Ustekinumab as induction and maintenance therapy for Crohn's disease. $N$ Engl J Med. 2016;375:1946-1960.

15. Feagan BG, Rutgeerts $\mathrm{P}$, Sands BE, et al. Vedolizumab as induction and maintenance therapy for ulcerative colitis. $N$ Engl J Med. 2013;369:699-710.

16. Colombel JF, Sandborn WJ, Reinisch W, et al. Infliximab, azathioprine, or combination therapy for Crohn's disease. $N$ Engl J Med. 2010;362:1383-1395.

17. Panaccione R, Ghosh S, Middleton S, et al. Combination therapy with infliximab and azathioprine is superior to monotherapy with either agent in ulcerative colitis. Gastroenterology. 2014;146:392-400.

18. Colombel JF, Adedokun OJ, Gasink C, et al. Combination therapy with infliximab and azathioprine improves infliximab pharmacokinetic features and efficacy: a post hoc analysis. Clin Gastroenterol Hepatol. 2019;17:1525-1532.

19. Vande Casteele N, Ferrante M, Van Assche G, et al. Trough concentrations of infliximab guide dosing for patients with inflammatory bowel disease. Gastroenterology. 2015;148:1320-1329.

20. Dreesen E, Baert F, Laharie D, et al. Monitoring a combination of calprotectin and infliximab identifies patients with mucosal healing of Crohn's disease. Clin Gastroenterol Hepatol. Epub. 05/22/2019.

21. Battat R, Kopylov U, Bessissow T, et al. Association between ustekinumab trough concentrations and clinical, biomarker, and endoscopic outcomes in patients with Crohn's disease. Clin Gastroenterol Hepatol. 2017;15:1427-1434.

22. Papamichael K, Chachu KA, Vajravelu RK, et al. Improved long-term outcomes of patients with inflammatory bowel disease receiving proactive compared with reactive monitoring of serum concentrations of infliximab. Clin Gastroenterol Hepatol. 2017;15:1580-1588.

23. Coutzac C, Chapuis J, Poullenot F, et al. Association between infliximab trough levels and the occurrence of paradoxical manifestations in patients with inflammatory bowel disease: a casecontrol study. J Crohns Colitis. 2015;9:982-987.

24. Seneschal J, Milpied B, Vergier B, Lepreux S, Schaeverbeke $\mathrm{T}$, Taieb A. Cytokine imbalance with increased production of interferon-alpha in psoriasiform eruptions associated with antitumour necrosis factor-alpha treatments. $\mathrm{Br} J$ Dermatol. 2009;161:1081-1088.

Publisher's Note Springer Nature remains neutral with regard to jurisdictional claims in published maps and institutional affiliations. 